\title{
Performance Analysis of Polarization Diversity for Indoor Scenarios at 41.4 GHz and $61.5 \mathrm{GHz}$
}

\author{
Ana Vazquez Alejos, Manuel Garcia Sanchez, and Iñigo Cuiñas \\ Department of Teoria de la Señal y Comunicaciones, University of Vigo, Campus Universitario Lagoas s/n, 36310 Vigo, Spain \\ Correspondence should be addressed to Ana Vazquez Alejos, analejos@uvigo.es
}

Received 25 October 2011; Revised 1 January 2012; Accepted 9 February 2012

Academic Editor: Krishnasamy Selvan

Copyright ( $) 2012$ Ana Vazquez Alejos et al. This is an open access article distributed under the Creative Commons Attribution License, which permits unrestricted use, distribution, and reproduction in any medium, provided the original work is properly cited.

We present the radio channel experiments conducted in indoor scenarios to test the performance of a dual orthogonal polarization scheme in the single-branch receiver end of a mobile system with a centre frequency of $41.4 \mathrm{GHz}$ and $61.5 \mathrm{GHz}$. The diversity gain has been obtained according to three main combination methods for an outage probability of 1\%: signal selection, equal gain combining, and maximal ratio combining. Diversity gain calculated ranges from 0.25 to $4.5 \mathrm{~dB}$, depending on the combination technique used, the radiation pattern, the scenario, and the frequency band. The spatial variation of the cross-polar discrimination factor (XPD) has been estimated. The XPD ranges from $31 \mathrm{~dB}$ to $34 \mathrm{~dB}$ at $41.4 \mathrm{GHz}$ and between $26 \mathrm{~dB}$ and $29 \mathrm{~dB}$ for the $61.5 \mathrm{GHz}$ band. From the gain and discrimination results, we evaluated the performance of this scheme to conclude its suitability as either a diversity technique for reducing signal fading, as the $41.4 \mathrm{GHz}$, or as a method for increasing the link capacity in the $61.5 \mathrm{GHz}$ case.

\section{Introduction}

Different diversity techniques have been reported in the literature [1-3]; nevertheless, the methods consisting of spatial and polarization diversity schemes appear as the most widely used choices to be implemented in mobile wireless communications. The polarization diversity scheme consists of receiving simultaneously the transmitted signals on orthogonally polarized waves with fade-independent characteristics. The diversity gain derived from the adopted combination scheme determines the viability of the system as an applicable diversity technique to provide signal fading mitigation.

On the other hand, the dual polarisation technique can also be used to increase the link capacity in terms of number of users that the system can support; this is achieved by providing channels in both polarizations in order to increase the system throughput. For that purpose, the cross-polar discrimination offered by the transmitted signals should have enough power range to counterpart the depolarization that the transmitted waves can undergo due to different propagation mechanisms or atmospheric phenomena, hydrometeors [4] being the main one.
In this paper, we introduce a narrowband system provided with a dual linear orthogonal polarization in order to determine the performance of this scheme as either an applicable diversity technique to provide signal fading mitigation or as a viable method to increase the number of users in the system. The measurement experiments were carried out at the centre frequency of $41.4 \mathrm{GHz}$ and $61.5 \mathrm{GHz}$ in an empty indoor scenario, and in the $41.4 \mathrm{GHz}$ case, an experiment was also conducted in a room with furniture. The transmitter and receiver antennas consisted of standard rectangular horns for both narrowband sounder ends. In the first indoor scenario, and only for the $61.5 \mathrm{GHz}$ case, we additionally tested an omnidirectional configuration in the receiver end, which could not be applied in the other cases due to insufficient dynamic range.

The spectrum region between $40.5 \mathrm{GHz}$ and $43.5 \mathrm{GHz}$ has been designated in Europe for Multimedia Wireless System $[5,6]$ to deploy point-to-multipoint fixed broadband wireless access (FBWA). Notwithstanding the possibilities offered by this band, it remains quite unknown and not extensively used in Europe $[6,7]$. The initial attention 


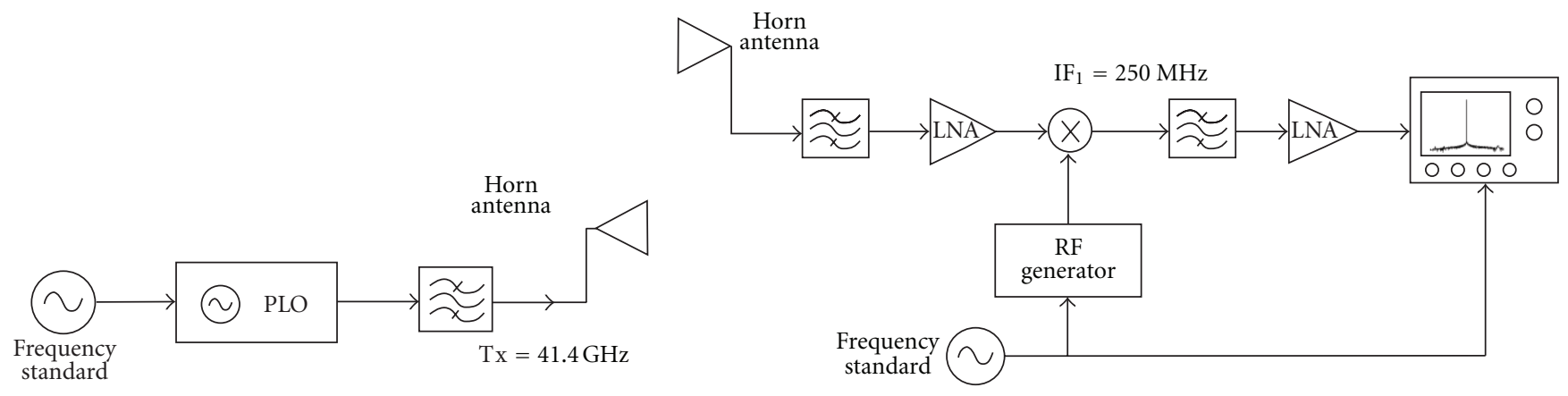

(a)

(b)

FIGURE 1: Functional diagram of narrowband sounder used at $41.4 \mathrm{GHz}$ experiments: (a) transmitter and (b) receiver.

disappeared and was sided by the emerging wireless communications networks operating at $2.4 / 5.8 \mathrm{GHz}$ and $60 \mathrm{GHz}$ bands, mainly due to their unlicensed feature.

The frequency band in the range of $57-64 \mathrm{GHz}$ has been allocated to deploy license-free wireless communications systems with Gigabit data rates that previously could only be accomplished with fibre optic cable [8]. Personal wearable applications are also contemplated in the latter frequency band, which has not been widely used either.

The polarization diversity has been contemplated in the regulations $[5,8]$ as a method to double the number of channels available in a radio communication link. Diversity techniques are also designed to mitigate impairments due interferences and propagation mechanisms. However, in both the $41.4 \mathrm{GHz}$ and $61.5 \mathrm{GHz}$ cases, experimental results regarding the performance of polarization diversity are scarce in the literature, especially for the millimetre frequency bands herein considered. Polarization diversity performance depends on the considered scenarios. So, while it works like space diversity in high-multipath environments such as dense urban $[1,2]$, it does not offer the same performance if used in an environment without severe multipath, such as the scenarios here presented.

Throughout the experiments presented in this paper we analyze the behaviour of the polarization diversity for the millimetre radio channel and indoor scenarios in both senses: as a diversity technique for reducing signal fading and as a method for increasing the link capacity.

In Section 2, we introduce the narrowband measurements conducted at $41.4 \mathrm{GHz}$. We describe the sounder utilized as well as the processing performed. The latter consisted of fitting the power decay law with the distance, the estimation of the cross-polar discrimination factor, the calculation of the diversity gain according to three signal combination methods (signal selection, equal gain combining, and maximal ratio combining), and an analysis of the fading correlation. In Section 3, we introduce the experiments carried out for the $61.5 \mathrm{GHz}$ case, with a similar processing. Finally, in Section 4 we offer the conclusions to this paper.

\section{Measurement Experiments at $41.4 \mathrm{GHz}$}

A narrowband sounder was built up according to the block diagram shown in Figure 1. The transmitter block is based
TABLE 1: Spectrum analyzer settings.

\begin{tabular}{lccc}
\hline Parameter & \multicolumn{2}{c}{ Value } & Units \\
\hline Frequency band & 61.5 & 41.4 & $\mathrm{GHz}$ \\
Receiver BW & 100 & 100 & $\mathrm{KHz}$ \\
Video BW & 100 & 100 & $\mathrm{KHz}$ \\
Center frequency & 20.1 & 0.25 & $\mathrm{GHz}$ \\
SPAN & 10 & 10 & $\mathrm{MHz}$ \\
Trace & 501 & 501 & points \\
Averaging & 50 & 25 & - \\
\hline
\end{tabular}

on a phase-locked oscillator (PLO) operating at $41.4 \mathrm{GHz}$. A pure tone of $+22 \mathrm{dBm}$ is transmitted by a pyramidal horn with $20 \mathrm{dBi}$ of gain and a beamwidth of $20^{\circ}$. The receiver side is based on a super heterodyne receiver consisting of a waveguide filter, a low-noise amplifier (LNA)- and a mixer. The mixer output is a carrier at an intermediate frequency of $250 \mathrm{MHz}$ that is recorded using a frequency spectrum analyzer, whose configuration is indicated in Table 1. The receiver was also provided with a horn antenna identical to the transmitter one, for the two indoor cases. Unlike in [1], the receiver used here is equipped with only one antenna and one superheterodyne branch. Both directional antennas could be rotated $90^{\circ}$ to work with vertical or horizontal polarization. Then, the four different polarization combinations were considered ( $\mathrm{HH}, \mathrm{HV}, \mathrm{VV}$, and $\mathrm{VH})$. These four measurements were taken consecutively, not simultaneously.

The narrowband sounder was tested in two rectangular indoor environments under quiescent conditions and without people in the room. This guaranteed that the propagation conditions were the same for the four consecutive measurements. The first scenario consisted of a classroom full of parallel rows of desks, sizing $25 \mathrm{~m}$ by $12 \mathrm{~m}$, with concrete walls and windows along three walls. The second scenario was an empty classroom, without any furniture, sizing $9.85 \mathrm{~m}$ by $8.76 \mathrm{~m}$, with walls made of brick and windows along one side wall. The separation distance between transmitter and receiver was $13.5-15.5 \mathrm{~m}$ for indoor case number 1 and $5.65-7.65 \mathrm{~m}$ for indoor case number 2 . The antenna height was $1.65 \mathrm{~m}$ and $1.52 \mathrm{~m}$, respectively, and in the classroom scenario the receiver antenna was placed on the central desks at a height of $0.65 \mathrm{~m}$. 


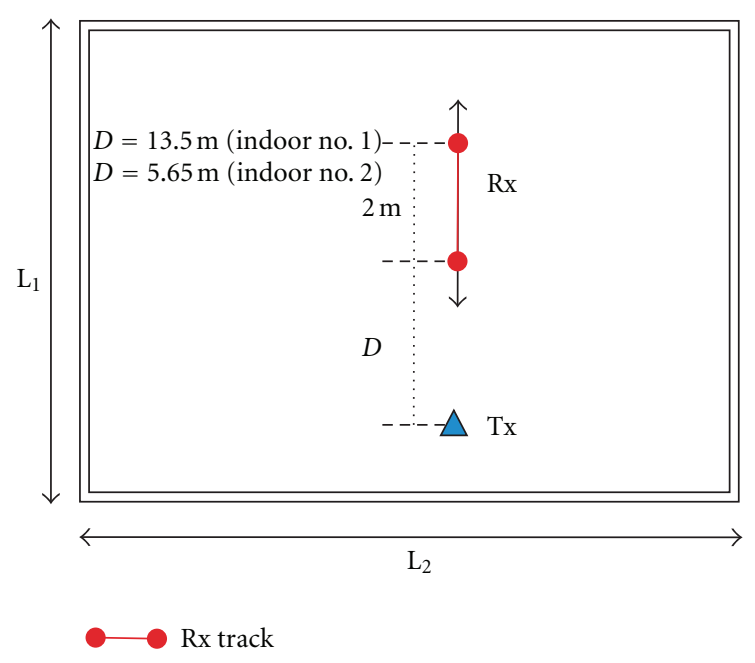

(a)

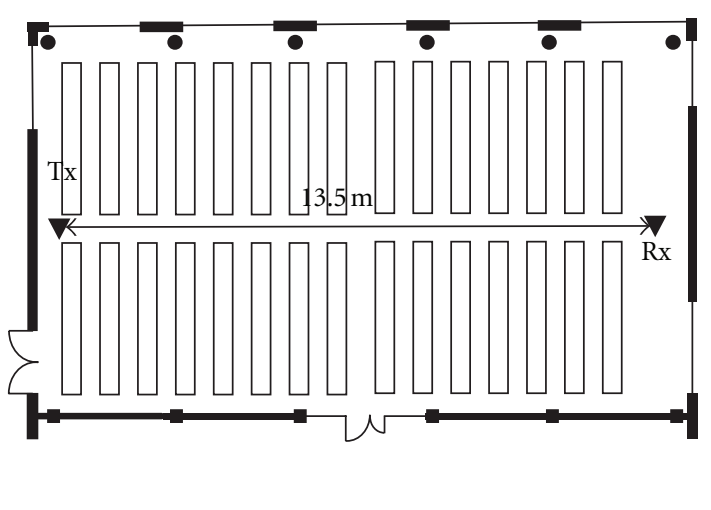

(b)

FIGURE 2: (a) geometry of indoor scenarios and relative placement of elements (transmitter, receiver, and linear track). (b) layout of indoor number 1 with details of furniture placement.

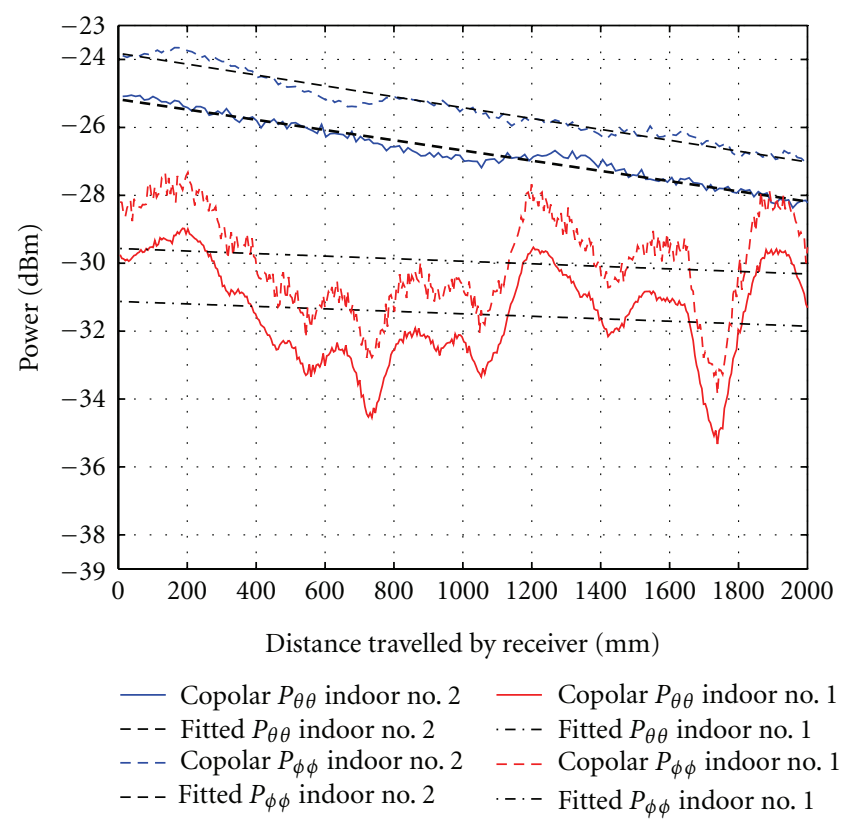

Figure 3: Power variation against distance of the copolar received signal envelope at $41.4 \mathrm{GHz}$ and power decay law fitting.

The narrowband sounder's receiver end is mobile, travelling along a linear track to cover a distance of $285 \lambda(2 \mathrm{~m})$ for both indoor cases number 1 and number 2 . The receiver displacement is controlled by a step-by-step motor to assure that the antenna was placed exactly at the same points. At the receiver end, the vertical and horizontal copolar ( VV or $e_{\theta \theta} ; \mathrm{HH}$ or $e_{\phi \phi}$ ) and cross-polar ( $\mathrm{VH}$ or $e_{\theta \phi}$ ) signal envelopes are consecutively registered in each $\lambda / 2(3.5 \mathrm{~mm})$ by means of the spectrum analyzer as indicated above. In Figure 2(a), we have illustrated the relative position of the measurement elements (transmitter, receiver, and linear track) in the indoor scenarios herein considered. Figure 2(b)

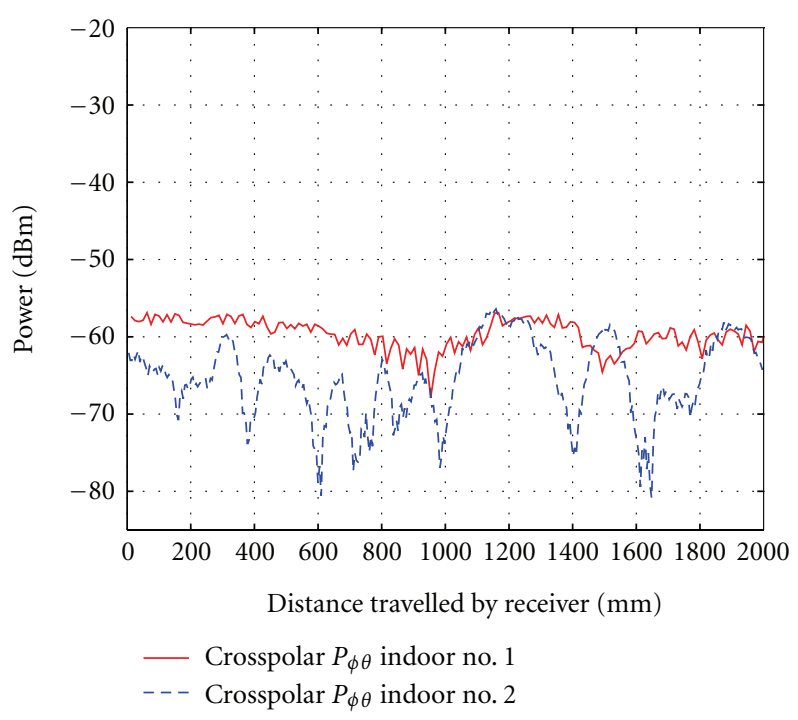

Figure 4: Power variation against distance of the cross-polar received signal envelope at $41.4 \mathrm{GHz}$.

shows a layout of the furniture placement for scenario indoor number 1 .

By this measurement setup, the polarization diversity could be evaluated with the receiver antenna at exactly the same positions, with no antenna separation. Consequently, the results correspond exclusively to the polarization diversity without any space diversity effect. Space diversity studies can be found in $[1,2]$. The configuration introduced in this paper represents a difference with other diversity studies, such as [9] where space and polarization diversity are combined.

The results for the spatial fading of the received signal envelopes for copolar and cross-polar components have been represented in Figure 3 and Figure 4, respectively. We can 
observe that a fast fading component is superimposed on the power decay, which is stronger for the furniture room case.

2.1. Signal Envelope Power Variation. The variation range of the received signal envelope is $6.5 \mathrm{~dB}$ and $3.25 \mathrm{~dB}$, for indoor environments number 1 and number 2, respectively. A larger fast fading was found in furniture-room case due to a stronger multipath situation.

The power decay has been fit for these measurements according to a log-distance model law, and the results are shown in Figure 3 and Figure 4. The fitting model is described by (1):

$$
P(d)=P_{0}+n \cdot d:(\mathrm{dBm}),
$$

where $P$ is the received power in $\mathrm{dBm}, P_{0}$ is the received power in $\mathrm{dBm}$ at a reference distance from the transmitter ( $13.5 \mathrm{~m}$ and $5.65 \mathrm{~m}$ for indoor number 1 and indoor number 2 , resp.), and $n$ is the power decay exponent in $\mathrm{dB} / \mathrm{m}$. The parameters $\left(n, P_{0}\right)$ found for the measurement scenarios were $(-0.3646,-31.12)$ for $P_{\theta \theta}$ indoor case number 1; $(-0.3757,-29.565)$ for $P_{\phi \phi}$ indoor case number $1 ;(-1.5053$, -25.17) for $P_{\theta \theta}$ indoor case number $2 ;(-1.6004,-23.81)$ for $P_{\phi \phi}$ indoor case number 2 .

2.2. Diversity Gain. In order to estimate the feasible diversity gain, we compare three signal combination methods widely reported in the literature $[1,2]$ : signal selection (SS) (2), equal gain combining (EGC) (3), and maximal ratio combining (MRC) (4). Given the signal envelopes in two orthogonal polarizations $e_{\theta \theta}$ and $e_{\phi \phi}$, the combined signal, $s$, according to these combination methods would be

$$
\begin{gathered}
s=\max \left\{e_{\theta \theta}, e_{\phi \phi}\right\}, \\
s=0.707 \cdot\left(e_{\theta \theta}+e_{\phi \phi}\right), \\
s=\sqrt{e_{\theta \theta}^{2}+e_{\phi \phi}^{2}} .
\end{gathered}
$$

We could also select a combination scheme of the received co- and cross-polar signals which is a possible implementation of a polarization diversity scheme, as in [10]; however, it would not have any sense here, given the high values of XPD as shown in Section 2.3.

The CDFs of the fast fading around the local mean of the different combined signals were calculated, and results are plotted in Figures 5 and 6, altogether with the original CDF of the measured copolar envelopes.

The CDFs were tested to follow a Rice distribution according to the $K-S$ test. Then, the $K$ factor was estimated according to [11]. For the indoor case number 1, the values found were $5.46(\mathrm{VV}), 4.96(\mathrm{HH}), 4.96$ (SS), 5.00 (EGC), and 4.98 (MRC). For the indoor case number 2 , the $K$ factor offered the values of 4.81 (VV), 5.02 (HH), 3.99 (SS), 5.75 (EGC), and 5.77 (MRC). The high $K$ values, mainly for indoor case number 2, confirm a weak multipath contribution compared to the one provided by the direct component. Due to the similarity of the $e_{\theta \theta}$ and $e_{\phi \phi}$ envelopes, it is reasonable to expect a comparable performance for the MRC

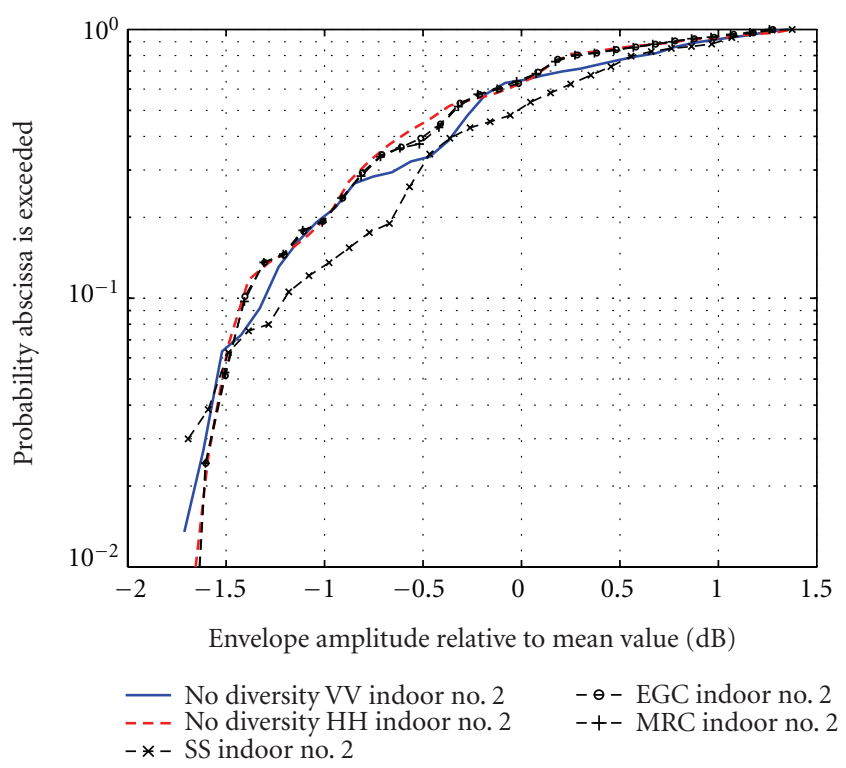

Figure 5: CDFs for different combination methods in indoor number 1 scenario at $41.4 \mathrm{GHz}$.

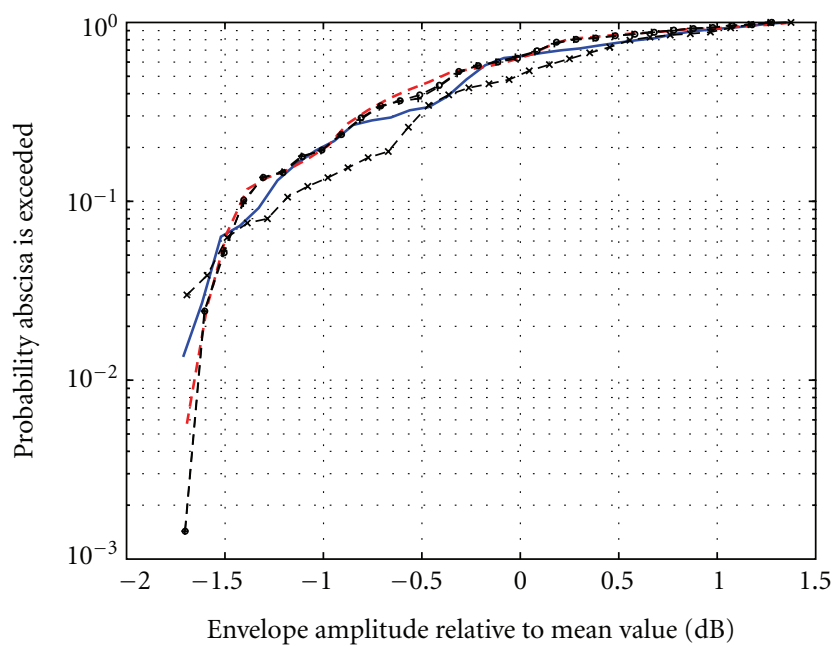

$\begin{array}{ll}\text { - No diversity VV indoor no. } 2 & -\bullet-\text { EGC indoor no. } 2 \\ --- \text { No diversity HH indoor no. } 2 & -+- \text { MRC indoor no. } 2 \\ -\star-\text { SS indoor no. } 2 & \end{array}$

FIGURE 6: CDFs for different combination methods in indoor number 2 scenario at $41.4 \mathrm{GHz}$.

and EGC combinations. The Rician $K$ factor values are summarized in Table 2 .

The performance of these combining techniques was quantified in terms of diversity gain for an outage of $1 \%$. For indoor case number 1 , none of the combination cases reduce the fading, due to the large fade correlation between the $e_{\theta \theta}$ and $e_{\phi \phi}$ envelopes. If the combination technique is SS, the diversity gain does not exist, resulting in $s$ equal to the signal registered in the $\mathrm{H}$-plane. For indoor case number 2 , the diversity gain is less than $0.25 \mathrm{~dB}$ if EGC or MRC are 
TABLe 2: Rician $K$ factor for the diversity gain CDFs.

\begin{tabular}{|c|c|c|c|c|c|c|}
\hline Frequency band & & $41.4 \mathrm{GH}$ & & & $1.5 \mathrm{GH}$ & \\
\hline Combination method & SS & EGC & MRC & SS & EGC & MRC \\
\hline $\begin{array}{l}\text { Indoor number 1: } \\
\text { directive }\end{array}$ & 4.96 & 5.0 & 4.98 & - & - & - \\
\hline Indoor number 2: omni & - & - & - & 4.66 & 3.18 & 3.30 \\
\hline $\begin{array}{l}\text { Indoor number } 2 \text { : } \\
\text { directive }\end{array}$ & 3.99 & 4.75 & 4.76 & 4.85 & 5.59 & 5.55 \\
\hline
\end{tabular}

used. This result also matches the low multipath present in the empty room scenario.

In terms of gain, the improvement achieved by the polarization diversity system at the receiver appears to be not remarkable due to the small signal fading. It appears also not noteworthy in comparison with spatial diversity gains that reach values around $5 \mathrm{~dB}$ as reported in $[1,2]$.

2.3. Cross-Polar Discrimination. The cross-polar component represents the depolarization suffered by the wave and is quantified by the cross-polar discrimination factor XPD $[10,12]$. The XPD is defined as the ratio of the average power of the incoming envelopes measured in orthogonal polarizations. For the vertical polarization, it is defined as in (5):

$$
\mathrm{XPD}_{\theta}=\frac{\left\langle\left|e_{\theta \theta}\right|^{2}\right\rangle}{\left\langle\left|e_{\theta \phi}\right|^{2}\right\rangle}=\frac{P_{\theta \theta}}{P_{\theta \phi}},
$$

and in a similar manner, it is given by (6) for the horizontal case:

$$
\mathrm{XPD}_{\phi}=\frac{\left\langle\left|e_{\phi \phi}\right|^{2}\right\rangle}{\left\langle\left|e_{\phi \theta}\right|^{2}\right\rangle}=\frac{P_{\phi \phi}}{P_{\phi \theta}} .
$$

The $\mathrm{XPD}_{\theta}$ is $31.69 \mathrm{~dB}$ and $32.95 \mathrm{~dB}$ for indoor number 1 and number 2 cases, respectively. The $\mathrm{XPD}_{\phi}$ is $33.89 \mathrm{~dB}$ and $34.21 \mathrm{~dB}$ for the same previous cases. In comparison with other values offered in the literature for mobile communications bands $[10,12]$, these values represent large crosspolar discrimination rate, and it would ensure the reliable operation of a system providing channels in orthogonal polarisations.

2.4. Power Fading Correlation. In Table 3, we summarize the fading correlation factors for the vertical and the horizontal polarizations: $\rho_{\theta \phi}$ and $\rho_{\phi \theta}$, respectively. These factors have been estimated as the correlation factor between the copolar $P_{i i}$ and cross-polar $P_{i j}$ power components, according to (7):

$$
\rho_{i, j}=\operatorname{corrcoef}\left(P_{i i}, P_{i j}\right),
$$

where the indexes $I$ and $j$ indicate copolar $(\theta \theta, \phi \phi)$ and cross-polar $(\theta \phi, \phi \theta)$ components, for vertical and horizontal polarisations.

Generally speaking, results shown in Table 3 indicate a not high correlation factor for $41.5 \mathrm{GHz}$. From results in
TABLE 3: Fading correlation factors.

\begin{tabular}{lcccc}
\hline Parameter & \multicolumn{2}{c}{$\rho_{\theta \phi}(\mathrm{VV}, \mathrm{VH})$} & \multicolumn{2}{c}{$\rho_{\phi \theta}(\mathrm{HH}, \mathrm{VH})$} \\
\hline $\begin{array}{l}\text { Frequency band } \\
\text { Indoor number 1: }\end{array}$ & $61.5 \mathrm{GHz}$ & $41.4 \mathrm{GHz}$ & $61.5 \mathrm{GHz}$ & $41.4 \mathrm{GHz}$ \\
directive & - & 0.459 & - & 0.438 \\
$\begin{array}{l}\text { Indoor number 2: } \\
\text { omni }\end{array}$ & 0.099 & - & 0.1249 & - \\
$\begin{array}{l}\text { Indoor number 2: } \\
\text { directive }\end{array}$ & 0.719 & 0.48 & 0.7503 & 0.399 \\
\hline
\end{tabular}

Table 3, for vertical polarization, we observe a slightly larger correlation factor for the directive indoor case number 2 than for the other cases indicating a lower depolarization in this scenario and case mainly due to the absence of multipath and other propagation impairments.

\section{Measurement Experiments at $61.5 \mathrm{GHz}$}

As shown in Figure 7, a similar narrowband measurement system was built up. The transmitter consisted of a RF signal generator that generated a $22.55 \mathrm{GHz}$ carrier. This signal was up converted to the $60 \mathrm{GHz}$ band by means of a mixer that was fed with an additional carrier at $38.95 \mathrm{GHz}$ given by a PLO. The $61.5 \mathrm{GHz}$ output signal with a power of $+20 \mathrm{dBm}$ was then transmitted by a $20 \mathrm{dBi}$ directional standard horn antenna.

The receiver end consisted of just one heterodyne branch system based on a LNA and a balanced mixer. The receiver antenna output boosted the LNA; then, using the same 41.4 GHz PLO than that of the system described in Section 2, the amplified RF signal was down converted to an intermediate frequency of $20.1 \mathrm{GHz}$, which was directly captured by a frequency spectrum analyzer. The analyzer main settings are summarized in Table 1 . Standard $10 \mathrm{MHz}$ frequency references were also utilized both in the transmitter and receiver ends to provide phase stability.

For the receiver antenna, we selected a $20 \mathrm{dBi}$ directional standard horn with a beamwidth of $20^{\circ}$, as in the transmitter end, and also a $2 \mathrm{dBi}$ omnidirectional antenna with a $60^{\circ}$ beamwidth in the vertical plane. For the horizontal polarization measurements, the omnidirectional antenna was rotated $90^{\circ}$ in a plane perpendicular to the line-of-sight (LoS) direction.

The measurement procedure was identical to the described in Section 2. The receiver travels along a linear track a distance of $410 \cdot \lambda(2 \mathrm{~m})$. However, only scenario number 2 was chosen for the experiments conducted at $61.5 \mathrm{GHz}$.

3.1. Power Variation of the Signal Envelope. In Figure 8, we observe that the variation range of the received signal envelope is $3.5 \mathrm{~dB}$ and $9 \mathrm{~dB}$, for directional receiver and for the omnidirectional case, respectively. The power decay with distance was fitted, and the results are shown in Figure 8. The power variation follows a linear distance model as in (1), with $P_{0}$ the received power in $\mathrm{dBm}$ at a reference distance of $5 \mathrm{~m}$ from the transmitter. The parameters $\left(n, P_{0}\right)$ found were 


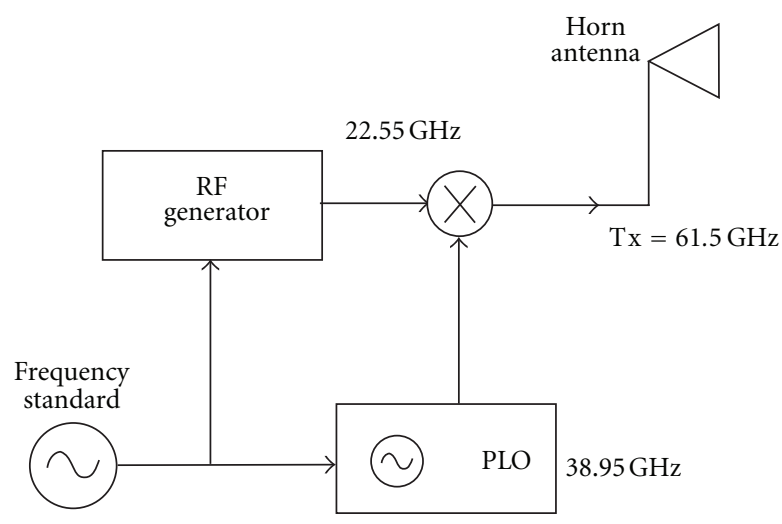

(a)

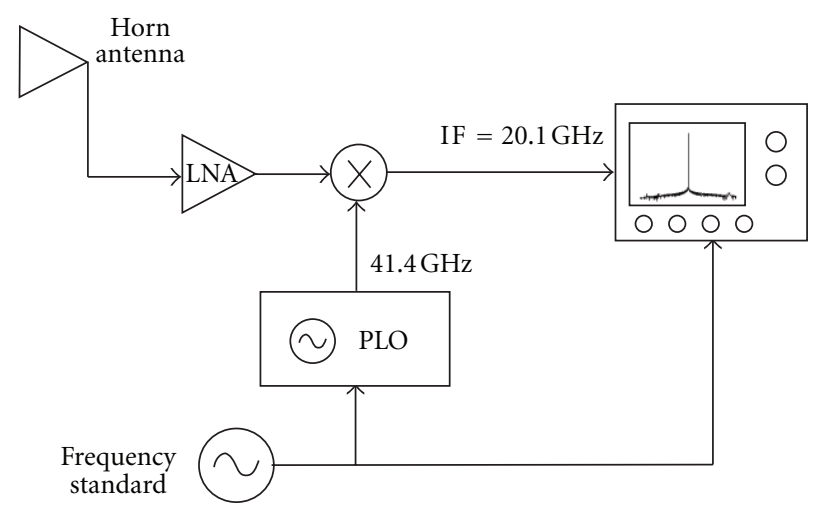

(b)

FiguRE 7: Functional diagram of narrowband sounder used at $61.5 \mathrm{GHz}$ experiments: (a) transmitter and (b) receiver.

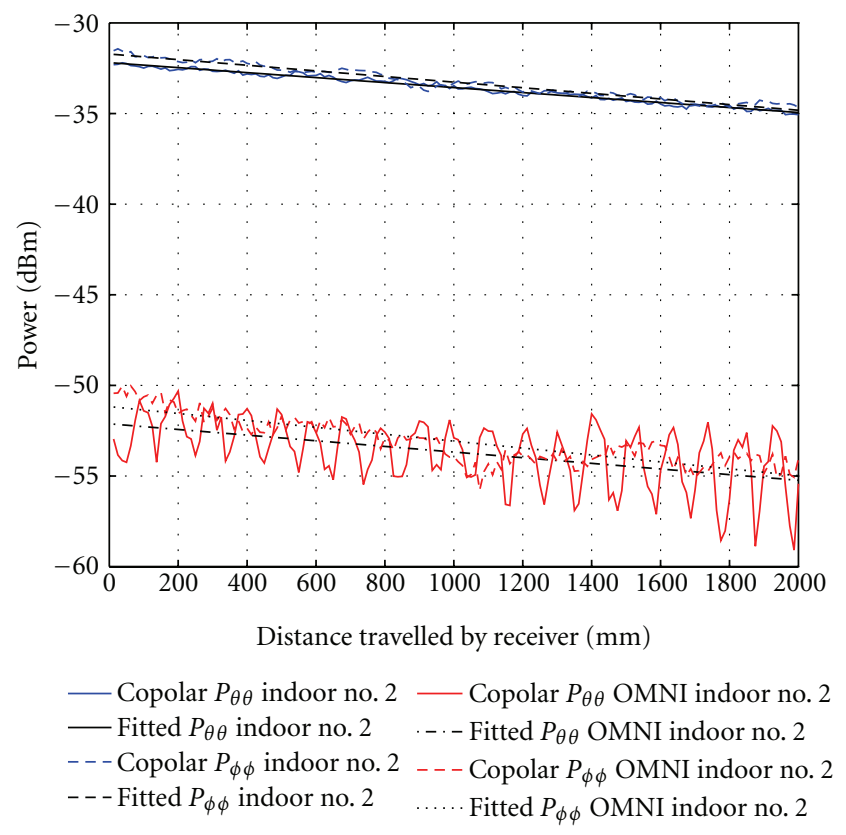

FIGURE 8: Power variation against distance of the copolar received signal envelope in indoor number 2 scenario at $61.5 \mathrm{GHz}$, and power decay law fitting.

$(-1.376,-25.31)$ for directional $P_{\theta \theta} ;(-1.549,-23.96)$ for directional $P_{\phi \phi} ;(-1.558,-44.33)$ for omnidirectional $P_{\theta \theta}$; $(-1.908,-41.62)$ for omnidirectional $P_{\phi \phi}$.

The resulting value of $n$ below the free space value, $n=2$, has also been reported in the literature at $60 \mathrm{GHz}$ for line-ofsight situations in a laboratory environment $[1,2,13]$ and in corridors [14], but also in other propagation experiments in the millimeter wave band [15].

3.2. Diversity Gain. The CDFs of the fast fading around the local mean for the three different combined signals (2)-(4) have been calculated, and results are plotted in Figures 9

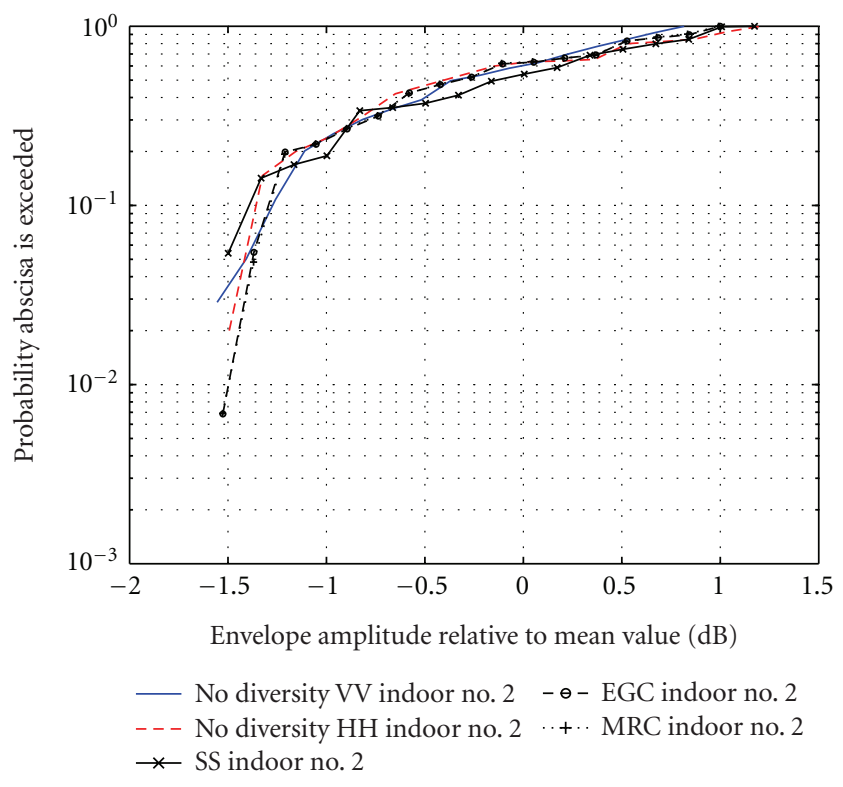

Figure 9: CDFs for different combination methods in indoor number 2 scenario at $61.5 \mathrm{GHz}$ and directional receiver.

and 10, as well as the CDF related to the original measured copolar envelopes $e_{\theta \theta}$ and $e_{\phi \phi}$.

For the $61.5 \mathrm{GHz}$ experiments, the CDFs also follow a Rice distribution. For the directional case, the values found for the Rician $K$ factor were 4.91 (VV), 6.27 (HH), 4.85 (SS), 5.59 (EGC), and 5.55 (MRC). For the omnidirectional case, the $K$ factor offered the values 2.77 (VV), $5.53(\mathrm{HH})$, 4.66 (SS), 3.30 (EGC), and 3.75 (MRC). The high $K$ values confirm the weak multipath compared to the direct contribution, mainly for the directional case. The Rician $K$ factor values are summarized in Table 2 .

We observe that for the directional case, the diversity gain is not remarkable due to the small signal fading and large fade correlation between the envelopes $e_{\theta \theta}$ and $e_{\phi \phi}$, as explained later in Section 3.4. 
However, for the omnidirectional case, the diversity gain is larger, especially if SS combination is used. In this case, the diversity gain is $4 \mathrm{~dB}$ that represents only a $+1 \mathrm{~dB}$ larger value than those achieved for EGC and MRC, in the best case that is versus the VV polarization. This value is comparable to the spatial diversity gain reported in $[1,2]$ that reaches values around $5 \mathrm{~dB}$. For the worst case, measured versus the $\mathrm{HH}$ curve, the diversity gain is less than $0.75 \mathrm{~dB}$ for EGC and MRC and null for SS.

3.3. Cross-Polar Discrimination. The cross-polar components are plotted in Figure 11 and summarized in Table 3. Using (5) and (6), the $\mathrm{XPD}_{\theta}$ results in $26.42 \mathrm{~dB}$ and $28.92 \mathrm{~dB}$ for directional and omnidirectional cases, respectively. The $\mathrm{XPD}_{\phi}$ is $26.82 \mathrm{~dB}$ and $29.52 \mathrm{~dB}$ in the same previous cases. As in the $41.4 \mathrm{GHz}$ experiments, these values represent large cross-polar discrimination indicating that the polarisation diversity would be more appropriate to provide orthogonal channels than to mitigate the fading.

3.4. Power Fading Correlation. Generally speaking, the values found for correlation factor at $61.5 \mathrm{GHz}$ are larger than for 41.4 GHz. The results presented in Table 3 show a significant difference between both antenna radiation patterns. The lowest factor $\rho$ is achieved for the omnidirectional case, for both vertical and horizontal polarisations, thus indicating a larger multipath situation for the scenario number 2 than in the directive antenna case.

\section{Conclusions}

Polarization diversity gain values are similar to the space diversity gain values achieved in high-multipath environments such as dense urban $[1,2,10,12,16]$. Consequently polarization diversity may be an alternative choice to space diversity, with the advantage of a more compact receiver configuration, given that the receiver antennas do not need to be separated a specific distance. However, it does not offer the same performance if it is used in an environment without severe multipath, such as the scenarios here presented.

The offered XPD values indicate a large fading correlation between copolar and cross-polar envelopes for the indoor scenarios. This fact together to the absence of depolarization mechanisms as hydrometeors indicates that the polarization diversity would not appear useful if used as a fading mitigation technique in indoor environments.

In the $41.4 \mathrm{GHz}$ case, the multipath has resulted larger in the furniture scenario, fact verified by the fading range and the Rician $K$ factor of the estimated CDF. But the influence of the multipath on the XPD is less significant, showing that the multipath does not affect severely to the cross-polar component. So we conclude that the dual polarization can be applied in indoor scenarios to increase the number of available channels better as a diversity technique to counterpart the fading mitigation.

For the $61.5 \mathrm{GHz}$ omnidirectional case, the outcomes are similar to those achieved at $41.4 \mathrm{GHz}$, so indicating that the technique can be useful to increase the capacity of

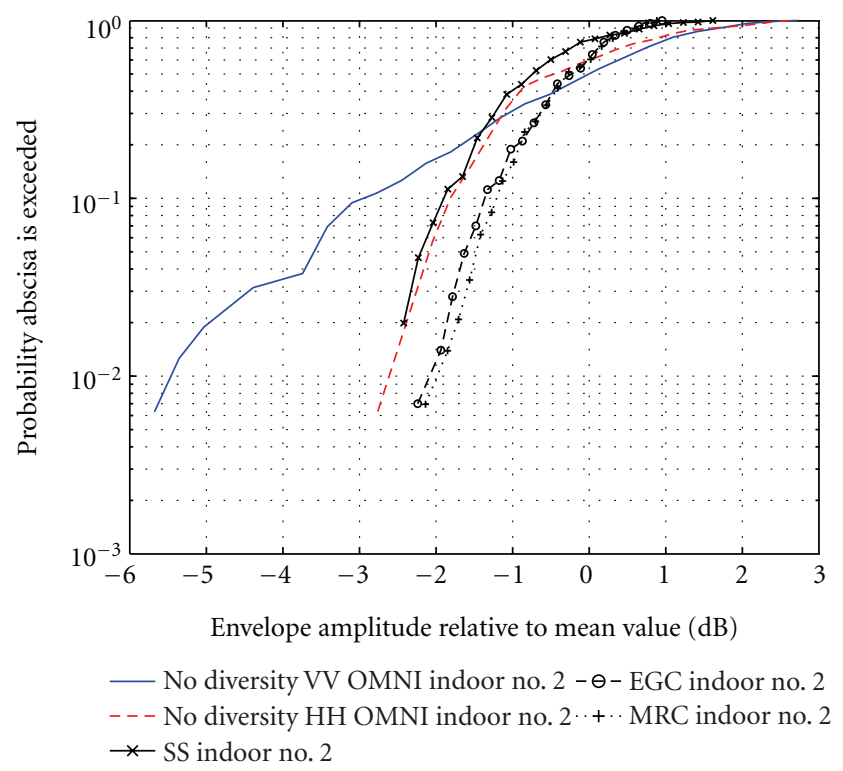

FIGURE 10: CDFs for different combination methods in indoor number 2 scenario at $61.5 \mathrm{GHz}$ and omnidirectional receiver.

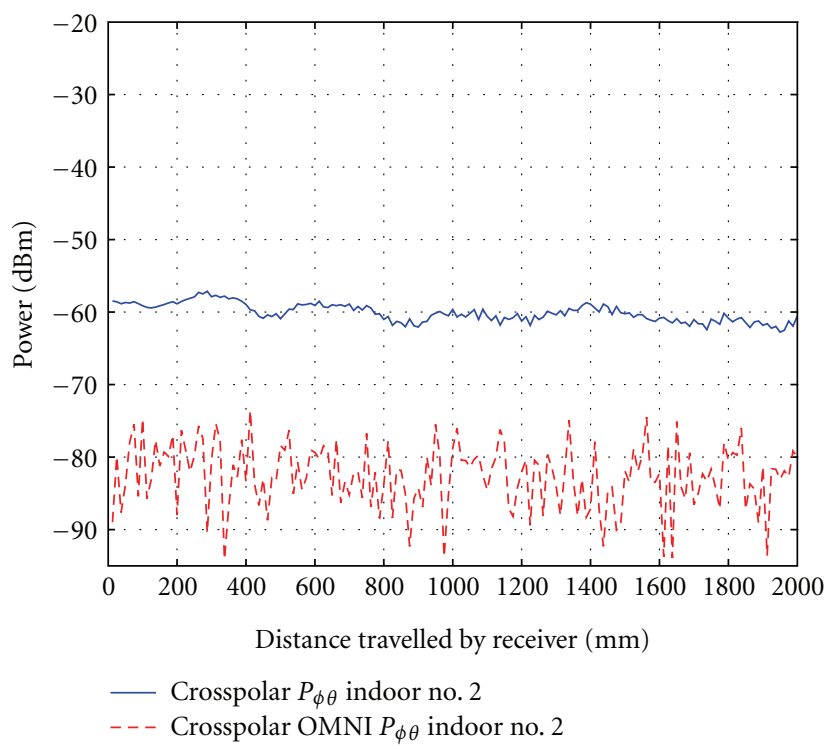

FIgURE 11: Power variation against distance of the cross-polar received signal envelope in indoor number 2 at $61.5 \mathrm{GHz}$.

the wireless communication system. However, the omnidirectional case indicates that the polarisation diversity can provide a gain comparable to that one given by the spatial diversity technique. In this case, it would be suitable to use the polarisation diversity as a fade mitigation technique than using dual polarization in order to double channel capacity. At the same time, the omnidirectional case shows a XPD value larger than that achieved for the directional case. This latter indicates that the omnidirectional configuration can be considered also to increase the number of channels.

The references in literature regarding experimental works on polarisation diversity are scarce, mainly in millimetric 
bands [6]. However, the results found in this paper agree with other reported in the literature, as aforementioned $[1,2,6]$. The explanation given for the values of power decay fitting factor $n$ below free space is the presence of wave-guiding effects in the propagation environments. Likewise, the Rician factor $K$ values agree with other found in the literature at millimeter wave frequencies under LoS conditions (e.g., [14]). Obviously, the averaging used in the measurement process turns the results here presented into values valid for averaged scenarios [10, p. 300].

The study of polarisation diversity for the 40 and $60 \mathrm{GHz}$ bands is important in order to achieve correct antenna design $[7,17]$, ray tracing, and simulators coverage [18-20], as well as to increase, or even double, the channel capacity [21].

Studies of diversity systems at other frequencies, as mobile frequency bands in [9] or millimetre wave band as described in [6], provide different results. This confirms the frequency and environment dependence of the diversity techniques performance.

\section{Acknowledgment}

This work was supported in part by the Ministerio de Ciencia e Innovación under Grant TEC2005-00330. The authors would also like to acknowledge the support of the People Program of 7th Framework Programme (2008 Marie Curie IOF Action).

\section{References}

[1] M. G. Sanchez, A. V. Alejos, and I. Cuiñas, "Space diversity performance in indoor radio channels at $40 \mathrm{GHz}$," IEE Electronics Letters, vol. 44, no. 20, pp. 1209-1210, 2008.

[2] M. G. Sanchez, A. V. Alejos, and I. Cuiñas, "Indoor radio reception at $60 \mathrm{GHz}$ : fading mitigation using diversity techniques," IEEE Transactions on Antennas and Propagation, vol. 57, no. 10, pp. 2934-2939, 2009.

[3] Z. Muhi-Eldeen, L. P. Ivrissimtzis, and M. O. Al-Nuaimi, "Measurements and modelling of cellular interference in local point-to-multipoint distribution systems," IET Microwaves, Antennas \& Propagation, vol. 3, no. 2, pp. 250-259, 2009.

[4] H. Fukuchi and J. Awaka, "Improved theoretical formula for the relationship between rain attenuation and depolarisation," IEE Electronics Letters, vol. 20, no. 21, pp. 859-860, 1984.

[5] CEPT Recommendation CEPT/ERC/REC(01)-04, Recommended Guidelines for the Accommodation and Assignment of Multimedia Wireless Systems (MWS) in the Frequency Band 40.5-43.5 GHz, 2001.

[6] A. V. Alejos, M. G. Sanchez, and I. Cuiñas, "Analysis of channel capacity for fixed broadband multimedia systems at $40 \mathrm{GHz}$," IET Microwaves, Antennas \& Propagation, vol. 5, no. 6, pp. 637-643, 2011.

[7] C. M. Tran, H. Hafdallah Ouslimani, L. Zhou et al., "High impedance surfaces based antennas for high data rate communications at $40 \mathrm{ghz}$," Progress in Electromagnetics Research C, vol. 13, pp. 217-229, 2010.

[8] C. Eklund, R. B. Marks, K. L. Stanwood, and S. Wang, "IEEE standard 802.16: a technical overview of the wirelessMAN air interface for broadband wireless access," IEEE Communications Magazine, vol. 40, no. 6, pp. 97-107, 2002.
[9] A. M. D. Turkmani, A. A. Arowojolu, P. A. Jefford, and C. J. Kellett, "Experimental evaluation of the performance of two-branch space and polarization diversity schemes at 1800 MHz," IEEE Transactions on Vehicular Technology, vol. 44, no. 2, pp. 318-326, 1995.

[10] R. G. Vaughan, "Polarization diversity in mobile communications," IEEE Transactions on Vehicular Technology, vol. 39, no. 3, pp. 177-186, 1990.

[11] A. Abdi, C. Tepedelenlioglu, M. Kaveh, and G. Giannakis, "On the estimation of the $\mathrm{K}$ parameter for the rice fading distribution," IEEE Communications Letters, vol. 5, no. 3, pp. 92-94, 2001.

[12] W. C. Y. Lee and Y. S. Yeh, "Polarisation diversity systems for mobile radio," IEEE Transactions on Communications, vol. 20, no. 5, pp. 912-923, 1972.

[13] N. Moraitis and P. Constantinou, "Indoor channel measurements and characterization at $60 \mathrm{GHz}$ for wireless local area network applications," IEEE Transactions on Antennas and Propagation, vol. 52, no. 12, pp. 3180-3189, 2004.

[14] G. A. Kalivas, M. El-Tanany, and S. Mahmoud, "Millimeterwave channel measurements with space diversity for indoor wireless communications," IEEE Transactions on Vehicular Technology, vol. 44, no. 3, pp. 494-505, 1995.

[15] A. Kajiwara, "Millimeter-wave indoor radio channel with artificial reflector," IEEE Transactions on Vehicular Technology, vol. 46, no. 2, pp. 486-493, 1997.

[16] P. Mattheijssen, M. H. A. J. Herben, G. Dolmans, and L. Leyten, "Antenna-pattern diversity versus space diversity for use at handhelds," IEEE Transactions on Vehicular Technology, vol. 53, no. 4, pp. 1035-1042, 2004.

[17] A. Reza Dastkhosh and H. Reza Dalili Oskouei, "A wideband high-gain dual-polarized slot array patch antenna for WiMAX applications in $5.8 \mathrm{GHz}$," International Journal of Antennas and Propagation, vol. 2012, Article ID 595290, 6 pages, 2012.

[18] T.-C. Tu, C.-M. Li, and C.-C. Chiu, "The performance of polarization diversity schemes in outdoor micro cells," Progress in Electromagnetics Research, vol. 55, pp. 175-188, 2005.

[19] T. Larbi and A. T. Denidni, "Ultrawideband measurements for wireless indoor communication systems at $37.2 \mathrm{GHz}$," Electronics Letters, vol. 38, no. 23, pp. 1477-1479, 2002.

[20] P. Combeau, L. Aveneau, R. Vauzelle, and Y. Pousset, "Efficient 2-D ray-tracing method for narrow and wideband channel characterisation in microcellular configurations," IEE Proceedings: Microwaves, Antennas and Propagation, vol. 153, no. 6, pp. 502-509, 2006.

[21] H. C. Lo, D. B. Lin, T.-C. Yang, and H.-J. Li, "Effect of polarization on the correlation and capacity of indoor MIMO channels," International Journal of Antennas and Propagation, vol. 2012, Article ID 246348, 11 pages, 2012. 

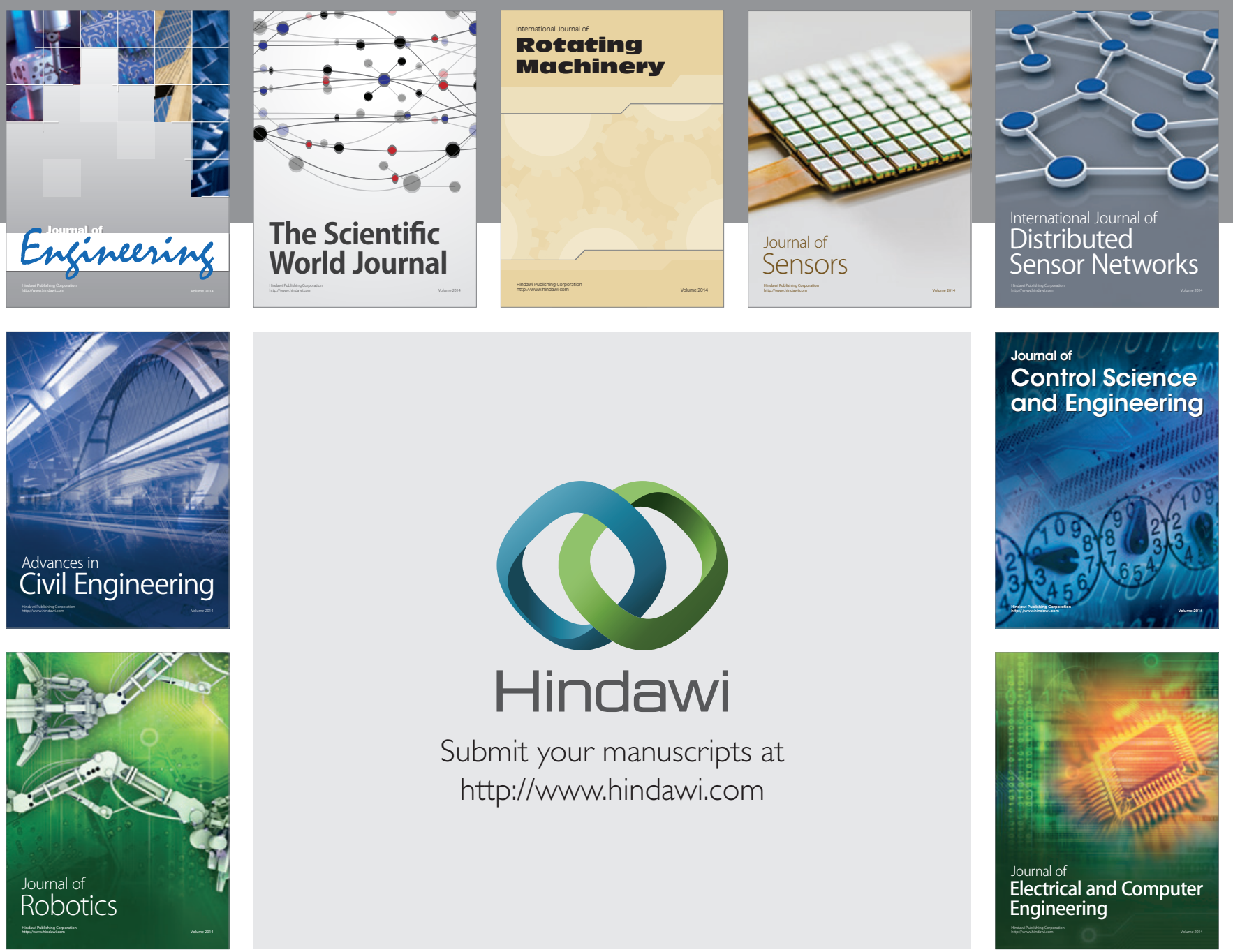

Submit your manuscripts at

http://www.hindawi.com
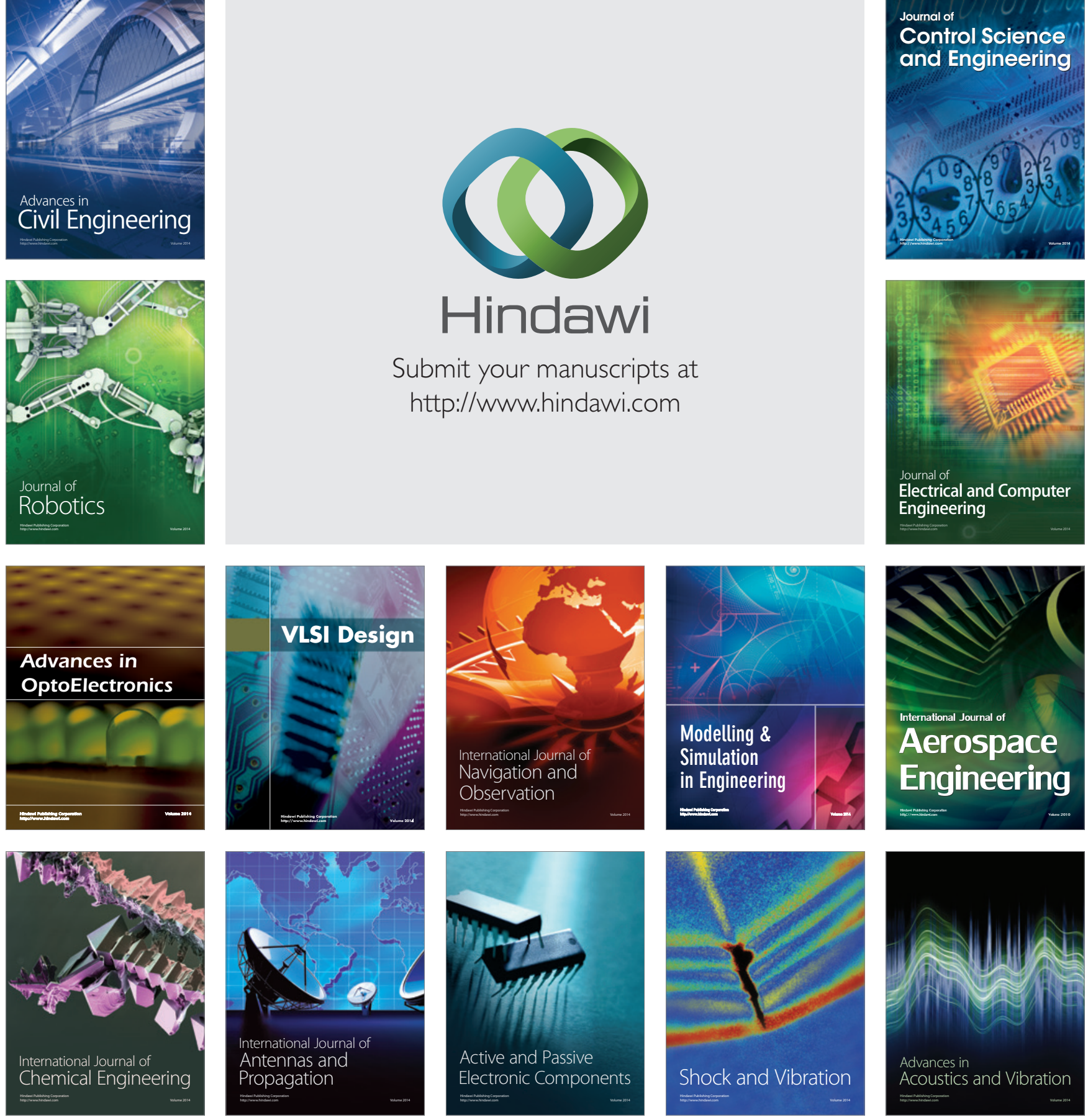